\title{
Flow of electro-rheological materials
}

\author{
K. R. Rajagopal, Pittsburgh, Pennsylvania, and A. S. Wineman, Ann Arbor, Michigan \\ (Received February 25, 1991)
}

\begin{abstract}
Summary. An electro-rheological fluid is a material in which a particulate solid is suspended in an electrically non-conducting fluid such as oil. On the application of an electric field, the viscosity and other material properties undergo dramatic and significant changes. In this paper, the particulate imbedded fluid is considered as a homogeneous continuum. It is assumed that the Cauchy stress depends on the velocity gradient and the electric field vector. A representation for the constitutive equation is developed using standard methods of continuum mechanics. The stress components are calculated for a shear flow in which the electric field vector is normal to the velocity vector. The model predicts (i) a viscosity which depends on the shear rate and electric field and (ii) normal stresses due to the interaction between the shear flow and the electric field. These expressions are used to study several fundamental shear flows: the flow between parallel plates, Couette flow, and flow in an eccentric rotating dise device. Detailed solutions are presented when the shear response is that of a Bingham fluid whose yield stress and viscosity depends on the electric field.
\end{abstract}

\section{Introduction}

During the past few years, there has been a great deal of interest in the manufacture and use of a class of materials which can be classified as field dependent rheological materials. These materials are essentially fluids which are imbedded with particulate solids which react to an electrical field in that on the application of a field the viscosity and other material properties undergo dramatic and significant changes. Such materials are being touted as agents for enhancing the performance and efficiency of a variety of engineering devices in very diverse fields. Much of the activity in this area is devoted to producing this material and performing experiments in order to understand the scientific basis for their behavior. Little, if any effort has been devoted to mathematically modeling these materials.

The need for understanding the mechanics of such materials and mathematically modeling their behavior is made all the more important as these materials are already finding day-to-day applications in the design of ubiquitous devices like clutches and brakes in cars, vibration dampers and absorbers, lubricating fluids in bearings to name some.

In this paper we shall present a mathematical model for field dependent materials which is consistent with the phenomena which have been observed. We shall solve a series of boundary value problems the results of which can be compared with future experiments, as these boundary value problems are in domains which are amenable to experimentation.

Unlike the field of magnetohydrodynamics, we do not have an equation like Maxwell's equation which governs the applied field, as the fluid which forms the base for the particulate media is non-conducting. The presence of the field alters the basic material properties of the particulate imbedded fluid, which is considered as a homogeneous continuum. Thus, for instance, the Cauchy stress is dependent on the gradient of the 
velocity and the electrical field. However, we need to consider only the usual balance laws of mass, momentum and energy. The electrical field enters as a parameter in the problem, which can be varied at will. The problem is also unlike classical magnetohydrodynamics in that the viscosity is a function of the electrical field.

In general the material properties would depend on the temperature. In fact, it could be possible to counter balance the effects of temperature by manipulating the electrical field. A case in point is the flow of such fluids in a journal bearing. Due to dissipation there is a great deal of heat generated in the flow domain and the temperature of the fluid rises considerably, resulting in a decrease in the viscosity of the fluid. Since it is necessary to maintain the load carrying capacity of the bearing, which depends on the viscosity, to be constant, a field dependent fluid accords the opportunity of achieving this goal. The electrical field is an additional parameter in the problem which can be adjusted towards this end.

We start our paper by deriving the constitutive relations for rheological materials in which the Cauchy stress depends on an electrical field and the velocity gradient of the fluid. Using standard arguments in continuum mechanics and representation theory we obtain representations for the stress when the material symmetry group is the proper orthogonal group and the full orthogonal group. Experiments seem to be consistent with the results obtained when the material symmetry group is the full orthogonal group, and thus our study shall be restricted to this model. For the purpose of illustration and in keeping with the indications of experiments, we shall restrict our analysis further to a simple class of constitutive models which exhibit the possibility of a yield stress. In this sense, the model resembles a Bingham material with a yield stress which is field dependent. Within the context of such a constitutive theory we study several boundary value problems, the results of which we hope can be compared with current and future experiments.

\section{Constitutive equation}

The mathematical model for the electro-rheological fluid is based on several assumptions. The first is that the fluid-suspended particle system can be represented as a continuum. The second is that the fluid responds instantaneously to changes in the electrical field. Thirdly, there are no history effects or long distance interactions in the response of the fluidparticulate system.

In order to present a mathematical statement of these assumptions, we introduce the following notation. Let $\boldsymbol{x}$ be a typical point in the continuum, $t$ the current time, $\boldsymbol{E}(\boldsymbol{x}, t)$ the electric field, $\boldsymbol{v}(\boldsymbol{x}, t)$ the velocity field, $\boldsymbol{L}=\operatorname{grad} \boldsymbol{v}$ the velocity gradient, and $\boldsymbol{T}$ the Cauchy stress teasor. The above assumptions lead to a constitutive equation of the form

$\boldsymbol{T}(\boldsymbol{x}, t)=\boldsymbol{T}[\boldsymbol{E}(\boldsymbol{x}, t), \boldsymbol{L}(\boldsymbol{x}, t)]$.

For the purpose of brevity, we drop further reference to $x$ and $t$ so that (1) becomes

$\boldsymbol{T}=\boldsymbol{T}(\boldsymbol{E}, \boldsymbol{L})$.

This constitutive equation must meet the restrictions imposed by material frame indifference (cf. Truesdell and Toupin [1]). The consequences of this restriction are that:

(a) the constitutive equation (2) can depend on $\boldsymbol{L}$ only through the stretching tensor $\boldsymbol{D}=1 / 2\left(\boldsymbol{L}+\boldsymbol{L}^{T}\right)$, and thus

$\boldsymbol{T}=\hat{\boldsymbol{T}}(\boldsymbol{E}, \boldsymbol{D})$, 
and

(b) the response function $\boldsymbol{T}$ must satisfy the relation

$\hat{\boldsymbol{T}}\left(\boldsymbol{Q E}, \boldsymbol{Q D} \boldsymbol{Q}^{T}\right)=\boldsymbol{Q} \hat{\boldsymbol{T}}(\boldsymbol{E}, \boldsymbol{D}) \boldsymbol{Q}^{T}$

where $Q$ is an orthogonal transformation, $Q Q^{T}=Q^{T} Q=I$, and (4) must hold for any choice of $\boldsymbol{E}$ and $\boldsymbol{D}$.

There has been some discussion in the literature as to whether $\boldsymbol{Q}$ can be only a rotation (an element of the proper orthogonal group of transformations with $\operatorname{det} Q=1$ ), or whether $Q$ could also be a reflection (an element of the full orthogonal group of transformations with $\operatorname{det} \boldsymbol{Q}=-1$ ). Here we shall give the representation corresponding to both these possibilities.

A method for determining the general form of the response function $\boldsymbol{T}(\boldsymbol{E}, \boldsymbol{D})$ which satisfies (4), for any group of orthogonal transformations, has been presented in the article by Spencer [2]. The results of applying this method are shown below for both the proper and full orthogonal group of transformations.

\subsection{Full orthogonal group}

If we require that (4) holds for any $\boldsymbol{Q}$ belonging to the full orthogonal group, then the constitutive equation has the representation

$$
\begin{aligned}
\boldsymbol{T}= & \alpha_{1} \mathbf{1}+\alpha_{2} \boldsymbol{E} \otimes \boldsymbol{E}+\alpha_{3} \boldsymbol{D}+\alpha_{4} \boldsymbol{D}^{2}+\alpha_{5}[\boldsymbol{D E} \otimes \boldsymbol{E}+\boldsymbol{E} \otimes \boldsymbol{D E}] \\
& +\alpha_{6}\left[\boldsymbol{D}^{2} \boldsymbol{E} \otimes \boldsymbol{E}+\boldsymbol{E} \otimes \boldsymbol{D}^{2} \boldsymbol{E}\right]
\end{aligned}
$$

in which $\alpha_{i}, i=1, \ldots, 6$ are scalar functions of the set of invariants

$I_{1}=\operatorname{tr}[\boldsymbol{E} \otimes \boldsymbol{E}], \quad I_{2}=\operatorname{tr} \boldsymbol{D}, \quad I_{3}=\operatorname{tr} \boldsymbol{D}^{2}$,

$I_{4}=\operatorname{tr} \boldsymbol{D}^{3}, \quad I_{5}=\operatorname{tr}[\boldsymbol{D E} \otimes \boldsymbol{E}], \quad I_{6}=\operatorname{tr}\left[\boldsymbol{D}^{2} \boldsymbol{E} \otimes \boldsymbol{E}\right]$.

\subsection{Proper orthogonal group}

If we assume that (4) ought to hold for any $\boldsymbol{Q}$ belonging to the proper orthogonal group, then the constitutive equation has the representation

$$
\begin{aligned}
\boldsymbol{T}= & \bar{\alpha}_{1} \mathbf{1}+\bar{\alpha}_{2} \boldsymbol{E} \otimes \boldsymbol{E}+\bar{\alpha}_{3} \boldsymbol{D}+\bar{\alpha}_{4} \boldsymbol{D}^{2}+\bar{\alpha}_{5}[\boldsymbol{A} \otimes \boldsymbol{E}+\boldsymbol{E} \otimes \boldsymbol{A}] \\
& +\bar{\alpha}_{6}[\boldsymbol{E} \otimes \boldsymbol{B}+\boldsymbol{B} \otimes \boldsymbol{E}]+\bar{\alpha}_{7}\left[\boldsymbol{M D}+\boldsymbol{D M} \boldsymbol{M}^{T}\right]+\bar{\alpha}_{8}\left[\boldsymbol{M} \boldsymbol{D}^{2}+\boldsymbol{D}^{2} \boldsymbol{M}^{T}\right] \\
& +\bar{\alpha}_{9}\left[\boldsymbol{D}^{2} \boldsymbol{M}^{T} \boldsymbol{D}+\boldsymbol{D M} \boldsymbol{D}^{2}\right]+\bar{\alpha}_{10}[\boldsymbol{E} \otimes \boldsymbol{M A}+\boldsymbol{M A} \otimes \boldsymbol{E}] \\
& +\bar{\alpha}_{11}[\boldsymbol{E} \otimes \boldsymbol{M B}+\boldsymbol{M B} \otimes \boldsymbol{E}]
\end{aligned}
$$

where the tensors $\boldsymbol{A}, \boldsymbol{B}$, and $\boldsymbol{M}$ are defined through:

$A_{i}=D_{i p} e_{p}, \quad B_{i}=D_{i p}^{2} e_{p}, \quad M_{i p}=\epsilon_{i p r} e_{r}$.

$\epsilon_{i j k}$ denotes the alternating tensor and $\bar{\alpha}_{i}, i=1,11$ are scalar functions of the set of invariants,

$I_{1}=\operatorname{tr}[\boldsymbol{E} \otimes \boldsymbol{E}], \quad I_{2}=\operatorname{tr} \boldsymbol{D}, \quad I_{3}=\operatorname{tr} \boldsymbol{D}^{2}, \quad I_{4}=\operatorname{tr} \boldsymbol{D}^{3}$,

$I_{5}=\operatorname{tr}[\boldsymbol{D E} \otimes \boldsymbol{E}], \quad I_{6}=\operatorname{tr}\left[\boldsymbol{D}^{2} \boldsymbol{E} \otimes \boldsymbol{E}\right]$,

$I_{7}=\operatorname{tr}\left[\boldsymbol{D}^{2} \boldsymbol{M}^{T} \boldsymbol{A} \otimes \boldsymbol{E}\right]-\operatorname{tr}\left[\boldsymbol{D}^{2} \boldsymbol{M}^{T} \boldsymbol{D}\right] I_{1}$. 
A number of comments can be made concerning the two forms of constitutive equations in (5) and (7). First, the scalar functions $\alpha_{i}$ and $\bar{\alpha}_{i}$ are material property functions, and are to be determined by experiments. Second, a comparison of the two constitutive equations shows that Eq. (7) contains Eq. (5) as a special case. In order to see this, note that Eq. (5) has the same form as the first six terms of Eq. (7). In addition, the set of arguments in (6) of the functions $\alpha_{i}$ are contained in the set of arguments (9) of functions $\bar{\alpha}_{i}$.

The second term in Eqs. (5) and (7) expresses the contribution of normal stress along the direction of the electric field vector. In order to see this, consider a surface area element whose unit normal vector $\boldsymbol{n}$ is parallel to $\boldsymbol{E}$, that is $\boldsymbol{n}=\lambda \boldsymbol{E}$. Since the stress vector on this area element is given by $\boldsymbol{T n}$, it follows that the contribution of the second term to the stress vector is $\alpha_{2} \hat{\lambda}|\boldsymbol{E}|^{2} \boldsymbol{E}$, which is normal to the surface element. The third and fourth terms represent the most general form of dependence on the tensor $\boldsymbol{D}$. The remaining terms account for the interaction between the rate of deformation tensor and the electric field vector.

The material response functions $\alpha_{i}$ and $\bar{\alpha}_{i}$ depend on the magnitude of the electric field through the invariant $I_{1}$. Invariants $I_{2}, I_{3}, I_{4}$ account for dependence on the rate of deformation tensor $\boldsymbol{D}$. The remaining invariants account for interaction between the deformation and electric fields.

Suppose that the fluid is incompressible. Then, the velocity field is subject to the constraint that tr $\boldsymbol{D}=D_{i i}=0$. The constitutive equations must then be modified to have the form

$\boldsymbol{T}=-p \mathbf{1}+\hat{\boldsymbol{T}}(\boldsymbol{E}, \boldsymbol{D})$,

in which $p$ denotes an arbitrary spherical stress which is superposed on the "extra stress" $\hat{\boldsymbol{T}}(\boldsymbol{E}, \boldsymbol{D})$. The form of $\hat{\boldsymbol{T}}(\boldsymbol{E}, \boldsymbol{D})$ is given by Eq. $(5)$, or Eq. (7), depending on whether the full or proper group of orthogonal transformations, respectively, is under consideration. Since $p$ is arbitrary, material property functions $\alpha_{1}$ and $\bar{\alpha}_{1}$ can be eliminated. In view of the constraint, $I_{2}=0$ and hence can be dropped from the list of invariants in (6) and (9).

Finally, note that each term in (5) and each invariant in (6) is of even degree in the components of $\boldsymbol{E}$. On the other hand, there are terms in (7) and an invariant in (9) which are of odd degree in the components of $\boldsymbol{E}$. Thus, under a reversal of the electric field, i.e., $\boldsymbol{E} \rightarrow-\boldsymbol{E}$, the stresses given by Eq. (5) will be unchanged whereas the stresses given by Eq. (7) will be altered.

\section{Shear flows}

We now consider the predictions of constitutive Eqs. (5) and (7) for shear flows in which the electric field vector is normal to the velocity vector. This is expected to represent a common situation which can arise in applications involving electro-rheological fluids.

The shear flow is assumed to occur in the $x_{1}-x_{2}$ plane of a Cartesian coordinate system. The form of the velocity field is then

$v=\gamma(t) x_{2} e_{1}$

and the electric field vector by

$\boldsymbol{E}=E_{2} e_{2}+E_{3} e_{3}$, 
where $\gamma(t)$ is the instantaneous shear rate and $\boldsymbol{e}_{1}, \boldsymbol{e}_{2}$ and $\boldsymbol{e}_{3}$ are the unit vectors in the $x_{1}, x_{2}$ and $x_{3}$ directions, respectively. For notational convenience, let $\gamma=\gamma(t)$. Consider first the predictions of constitutive Eq. (5). The stress components are found to be

$T_{11}=\alpha_{1}+\frac{\alpha_{4}}{4} \gamma^{2}$

$T_{22}=\alpha_{1}+\alpha_{2} E_{2}^{2}+\frac{\alpha_{4}}{4} \gamma^{2}+\frac{\alpha_{6}}{2} \gamma^{2} E_{2}^{2}$,

$T_{33}=\alpha_{1}+\alpha_{2} E_{3}^{2}$,

$T_{12}=\left(\frac{\alpha_{3}}{2}+\frac{\alpha_{5}}{2} E_{2}^{2}\right) \gamma$

$T_{13}=\frac{\alpha_{5}}{2} \gamma E_{2} E_{3}, \quad T_{23}=\alpha_{2} E_{2} E_{3}+\frac{\alpha_{6}}{4} \gamma^{2} E_{2} E_{3}$,

and by Eq. (6) the invariants are

$$
\begin{aligned}
& I_{1}=E_{2}{ }^{2}+E_{3}{ }^{2}, \quad I_{2}=0, \quad I_{3}=\frac{1}{2} \gamma^{2}, \quad I_{1}=0, \\
& I_{5}=0, \quad I_{6}=\frac{1}{4} \gamma^{2} E_{2}^{2} .
\end{aligned}
$$

Consider first the effect of the electric field component $E_{3}$. The in-plane stress components $T_{11}, T_{22}, T_{12}$ depend on $E_{3}$ only through the dependence of the functions $\alpha_{i}$ on $I_{1}$, the magnitude of the electric field vector. The most significant effect of $E_{3}$ is that it induces shear stress components $T_{13}$ and $T_{23}$. If these stresses are non-zero, there could also be shear flow in the $x_{1}-x_{3}$ and $x_{2}-x_{3}$ planes. This component also contributes to the normal stress.

In the remainder of this discussion, we shall assume that $E_{3}=0$. It follows that $T_{13}$ $=T_{23}=0$, and $I_{1}=E_{2}{ }^{2}$. For notational convenience, let $E_{2}=E$. It is seen from (14) that $\alpha_{i}$ can be regarded as functions of $E^{2}$ and $\gamma^{2}$. The shear stress-shear rate equation can then be written in the form

$T_{12}=\mu\left(E^{2}, \gamma^{2}\right) \gamma$

where

$\mu\left(E^{2}, \gamma^{2}\right)=\frac{1}{2}\left(\alpha_{3}+\alpha_{5} E^{2}\right)$.

$\mu\left(E^{2}, \gamma^{2}\right)$ represents the shear viscosity function which is seen to depend on both the electric field and the shear rate. The term $\alpha_{3}$ is the one which can account for the non-Newtonian nature of the fluid. The term in $\alpha_{5}$ arises from the interaction of the shear flow and the component of the electric field in the direction normal to the flow direction. When the electric field vanishes, so does this term and the viscosity reduces to a function of the shear rate, as is the case when we have the purely mechanical problem.

As seen in Eq. (13), the electric field gives rise to two terms in the expression for normal stress $T_{\mathbf{2 2}}$. There is thus a normal stress effect which is in addition to that arising from nonlinearities in the rate of deformation tensor. In the absence of an electric field, there are two equal normal stresses. Thus, the electric field creates three distinct normal stresses. In the case of an incompressible fluid, there are two distinct normal stress differences. 
We present next the predictions of constitutive Eq. (7). Because of the complexity of the terms in this equation, we shall consider only the case in which $E_{1}=E_{3}=0$. The stress components are found to be

$$
\begin{aligned}
& T_{11}=\bar{\alpha}_{1}+\frac{1}{4} \bar{\alpha}_{4} \gamma^{2} \\
& T_{22}=\bar{\alpha}_{1}+\bar{\alpha}_{2} E^{2}+\frac{1}{4} \bar{\alpha}_{4} \gamma^{2}+\frac{1}{2} \bar{\alpha}_{6} \gamma^{2} E^{2} \\
& T_{33}=\bar{\alpha}_{1} \\
& T_{12}=\frac{1}{2}\left(\bar{\alpha}_{3}+\bar{\alpha}_{5} E^{2}\right) \gamma \\
& T_{13}=\bar{\alpha}_{8} E \gamma^{2}, \\
& T_{23}=\bar{\alpha}_{7} E \gamma+\frac{1}{2} \bar{\alpha}_{10} \gamma E^{3} .
\end{aligned}
$$

Invariants $I_{1}$ through $I_{6}$ coincide with those in (14). It is found that $I_{7}=0$.

Stress components $T_{11}, T_{22}, T_{33}, T_{12}$ have the same form as those given by Eq. (5). Terms in $\bar{\alpha}_{7}-\bar{\alpha}_{11}$ give rise to the shear stress components $T_{13}, T_{23}$. No terms involving the material functions $\bar{\alpha}_{9}$ and $\bar{\alpha}_{11}$ are present because they mutiply quantities which are identically zero.

The results of experiments (cf. Filisko [3]) are consistent with the predictions of the constitutive equation (5). Hence, we shall consider only this representation in the remainder of this paper.

\subsection{Flows between parallel plates}

The fluid is confined between parallel plates which are a distance $2 h$ apart. The electric field vector is constant in both space and time, and acts in a direction perpendicular to the plates. The fluid flow is assumed to be rectilinear in a direction parallel to the plates. The flow is maintained by the combined effect of a constant body force and pressure gradient. As indicated in Fig. 1, the flow is referred to a Cartesian coordinate system which has its origin on the midsurface, the $x_{1}$ axis in the direction of flow, and the $x_{2}$ axis perpendicular to the plates.

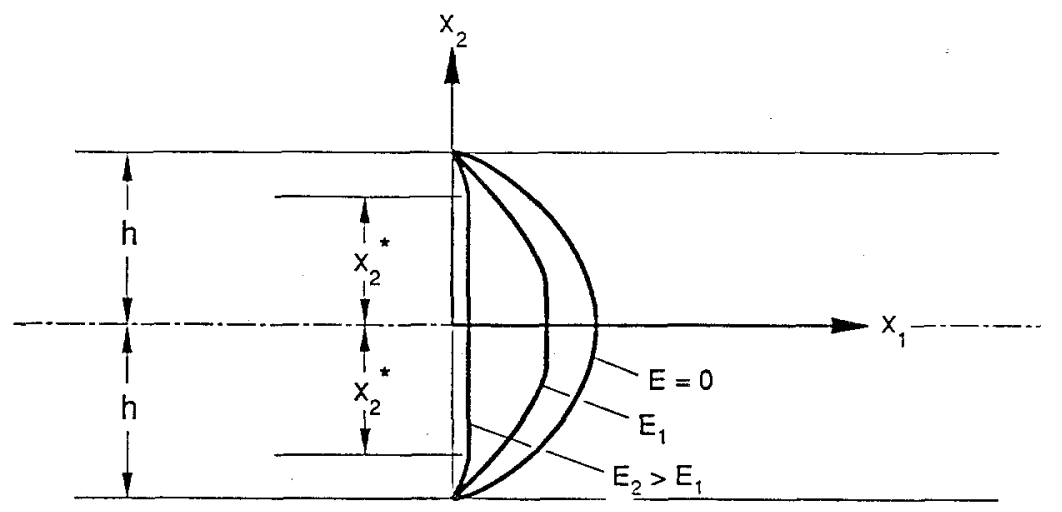

Fig. 1. Velocity profiles for flow between parallel plates. The pressure gradient is fixed and the electric field $E$ is increasing 
Let $\boldsymbol{b}$ denote the external body force per unit mass on the fluid. The body force, velocity, and electric fields are assumed to have the form

$\boldsymbol{b}=\varrho b \boldsymbol{e}_{1}$,

$v=v\left(x_{2}\right) e_{1}$

$\boldsymbol{E}=E e_{2}$,

where $b$ and $E$ are constant. By Eqs. (5) and (10), the stress components are

$T_{11}=-p+\frac{\alpha_{4}}{4} \gamma^{2}$,

$T_{22}=-p+\alpha_{2} E^{2}+\frac{\alpha_{4}}{4} \gamma^{2}+\frac{\alpha_{6}}{2} \gamma^{2} E^{2}$,

$T_{33}=-p$,

$T_{12}=\frac{1}{2}\left(\alpha_{3}+\alpha_{5} E^{2}\right) \gamma=\mu\left(E^{2}, \gamma^{2}\right) \gamma$,

$T_{13}=T_{23}=0$

in which $\gamma=d v / d x_{2}$, and $p$ is an indeterminate scalar which is yet to be determined.

The equations of motion must be satisfied for each fixed choice of $E$. Let the velocity field, body force and stresses be substituted into the equations of motion stated with respect to Cartesian coordinates. The form of the velocity field in (18) is such that the acceleration terms are identically zero. By standard arguments it can be shown that $T_{12}$ is an odd function of $x_{2}$, and thus the shear stress field is given by

$T_{12}=-A x_{2}, \quad A=P+b$,

in which $P$ is the pressure gradient, and the scalar $p$ is given by

$p=-P x_{1}+\alpha_{2} E+\frac{\alpha_{4}}{4} \gamma^{2}+\frac{\alpha_{6}}{2} \gamma^{2} E^{2}$.

On substituting the expression for shear stress $T_{12}$ from (19) into $\mathrm{Eq}$. (20), a differential equation is obtained for $v\left(x_{2}\right)$ which contains the parameters $E$ and $A$.

The velocity field must also satisfy the no-slip boundary conditions

$v(h)=v(-h)=0$.

Now consider a specific choice for the relation between $T_{12}, E$ and $\gamma$. There is some experimental evidence (cf. Filisko [3]) that suggests a relation of the form ${ }^{1}$ (see Fig. 2),

$T_{12}=\left\{\begin{array}{cc}\sigma_{0}(E)+\mu(E) \gamma, & \gamma>0 \\ 0, & \gamma=0 \\ -\sigma_{0}(E)+\mu(E) \gamma, & \gamma<0,\end{array}\right.$

1 In general, we could expect non-Newtonian fluid behavior even in the absence of an electric field, and thus $T_{12}$ might have the form:

$T_{12}=\sigma_{0}(E)+\mu\left(E^{2}, \gamma^{2}\right) \gamma$

where $\mu(., \gamma)$ is a non-linear function of $\gamma$. We could thus allow for the non-Newtonian behavior of the material even in the absence of an electrical field 


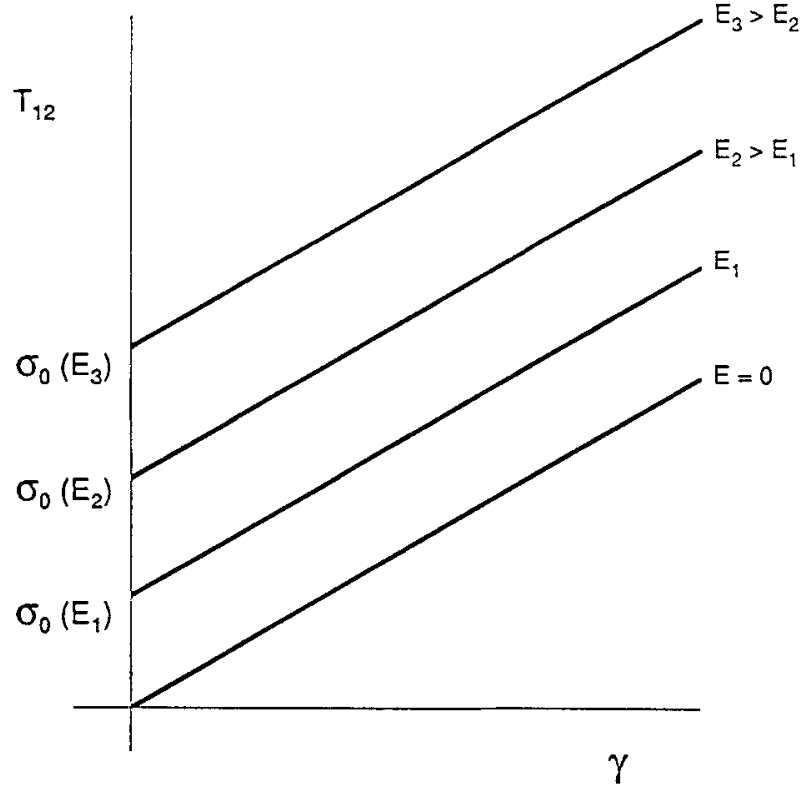

Fig. 2. A shear stress-shear rate relation for increasing electric field $E$

where $\sigma_{0}(0)=0$ and $\sigma_{0}(E)$ and $\mu(E)$ increase monotonically with $E$. According to Eq. (23), when $E=0$, the shear response is that of a classical linearly viseous fluid. When $E \neq 0$, the response becomes similar to that of a Bingham material, with yield stress $\sigma_{0}(E)$.

Now consider the determination of $v\left(x_{2}\right)$ for this model. For each fixed value of $E$, the maximum magnitude of shear stress occurs at the plates, $x_{2}= \pm h$, and has the value $A h$. If $A$ is such that $A \leqq \sigma_{0}(E)$, the magnitude of the shear stress is less than the yield stress at all points between the plates, and there is no flow. Let $A$ have a value such that $A>\sigma_{0}(E)$. There is some coordinate $x_{2}=x^{*}(E)$ at which $A x^{*}(E)=\sigma_{0}(E)$, i.e., at which the yield condition is satisfied. Then, according to Eqs. (20) and (23), the velocity field satisfies the differential equations

$$
\begin{aligned}
& -\sigma_{0}(E)+\mu(E) \frac{d v}{d x_{2}}=-A x_{2}, \quad x^{*}(E) \leqq x_{2} \leqq h \\
& \frac{d v}{d x_{2}}=0, \quad-x^{*}(E) \leqq x_{2} \leqq x^{*}(E) \\
& \sigma_{0}(E)+\mu(E) \frac{d v}{d x_{2}}=-A x_{2}, \quad-h \leqq x_{2} \leqq-x^{*}(E) ;
\end{aligned}
$$

When these are solved subject to the no-slip conditions (22) and the boundary conditions at $x_{2}= \pm h$, it is found that

$$
\begin{aligned}
& v\left(x_{2}\right)=\frac{1}{\mu(E)}\left[\frac{A}{2}\left(h^{2}-x_{2}{ }^{2}\right)-\sigma_{0}(E)\left(h-x_{2}\right)\right], \quad x^{*}(E) \leqq x_{2} \leqq h \\
& v\left(x_{2}\right)=\frac{1}{\mu(E)}\left[\frac{A}{2}\left(h^{2}-x^{*}(E)\right)-\sigma_{0}(E)\left(h-x^{*}(E)\right)\right] \\
& v\left(x_{2}\right)=\left(A h-\sigma_{0}(E)\right)^{2} / 2 A \mu(E), \quad-x^{*}(E) \leqq x_{2} \leqq x^{*}(E) \\
& v\left(x_{2}\right)=\frac{1}{\mu(E)}\left[\frac{A}{2}\left(h^{2}-x_{2}{ }^{2}\right)-\sigma_{0}(E)\left(h+x_{2}\right)\right], \quad-h \leqq x_{2} \leqq-x^{*}(E) .
\end{aligned}
$$


These equations describe a flow consisting of a central region in which the fluid moves as a rigid body, i.e., "plug flow", and outer layers in which the velocity profile is parabolic. If the shear viscosity $\mu\left(E^{2}, \gamma^{2}\right)$ is given by the more general expression in the footnote, then the flow field outside the dead zone or plug flow region would not be parabolic, but would correspond to that for an appropriate power-law non-Newtonian fluid.

Now consider the process in which $A$ is held fixed and the magnitude $E$ of the electric field increases. When $E=0$, then $\sigma_{0}(0)=0, x^{*}(E)=0$ and the velocity field reduces to the well known form for that of a Newtonian fluid in plane Poiseuille flow. As $E, \sigma_{0}(E)$, and $x^{*}(E)$ increase, it is seen from Eq. (25) that the velocity profile becomes that of plug flow. The thickness of the "plug" region increases and the maximum velocity decreases (see Fig. 1). When $E$ is such that $A h=\sigma_{0}(E)$, the maximum velocity reduces to zero. This corresponds to the fact that, by Eq. (20), the stress is everywhere less than the yield stress.

If the fluid is a shear thickening non-Newtonian power-law fluid increasing the shear rate and the electrical field both tend to increase the viscosity and the flow has a larger region of plug flow. However, if the fluid is shear thinning, increasing the shear rate and the electrical field have opposing effects and tend to counteract each other. This phenomenon has tremendous potential implications, for example in problems where the shear stresses induced in the fluid have to be nearly constant, a flow in a bearing being one example. It is well known that the viscosity of a fluid is temperature dependent and tends to decrease with increasing temperature. Such temperature rises occur due to the dissipation in the fluid. We can compensate for such changes in the viscosity by appropriately modifying the electrical field.

\subsection{A Rabinowitch-Mooney type relationship}

The general theory for the flow between parallel plates, presented in Eqs. (18)-(22), can be used to develop a Rabinowitch-Mooney type relation for determining the $T_{12}-E-\gamma$ relation from mass flow-pressure gradient measurements. Let $v\left(x_{2}, E\right)$ denote the velocity field and let $Q(E)$ denote the volumetric flow per unit plate width, for any value of $E$. These are related by

$Q(E)=\int_{-h}^{h} v\left(x_{2}, E\right) d x_{2}$.

Let it be further assumed that the shear stress-shear rate relation in Eq. (19.4) can be inverted so that

$\gamma=\gamma\left(T_{12}, E\right)$.

This is quite a strong assumption, though valid for many real rheological materials. However, there are materials where this assumption does not hold and much of the recent work on materials which allow for phase-transformations within the context of elastic and viscoelastic materials has as its basis the non-invertibility of relations such as (19).

On integrating the right hand side of Eq. (26) by parts, using the adherence conditions (22), and then the relation $\gamma=d v / d x_{2}$ the expression for the mass flux can be written as

$Q=-\int_{-h}^{h} x_{2} \gamma\left(T_{12}\left(x_{2}\right), E\right) d x_{2}$. 
Now consider the change of variable implied by Eq. (20),

$x_{2}=-\frac{\sigma}{A}$.

Using the fact that $T_{12}$ is an odd function of $\gamma$ and the invertibility, Eq. (28), can be rewritten in the form

$\frac{Q}{h^{2}}=\frac{1}{B^{2}} \int_{-A h}^{A h} \sigma \gamma(\sigma, E) d \sigma$

in which $B=A h$ denotes the wall shear stress, and use has been made of symmetry about the $x_{1}$-axis. Finally taking the derivative with respect to $B$ in Eq. (30), we obtain the desired result,

$B \gamma(B, E)=\frac{1}{2 h^{2}} \frac{d}{d B}\left(Q B^{2}\right)$

and hence

$\gamma(B, E)=\left(\frac{1}{2 h^{2}}\right)\left(2 Q+B \frac{d Q}{d B}\right)$.

Thus, suppose that the volume rate of flow-pressure gradient graph has been determined for some fixed value of $E$. Then, the relation between the shear rate at the wall and shear stress at the wall can be constructed by means of Eq. (31).

\subsection{Couette flow}

Next, suppose that the incompressible electro-rheological fluid is contained between two concentric cylinders. The inner and outer radii of the fluid region are $R_{i}$ and $R_{0}$, respectively. No body force acts on the fluid. The electric field vector is radial and its magnitude is constant. The outer cylinder is held fixed and a moment is applied to the inner cylinder about its axis. The velocity field is assumed to be in the circumferential direction and varies only with radius. Accordingly, there is a local shearing flow in the circumferential direction, with the electric field vector in a direction normal to the velocity field.

The components, with respect to cylindrical coordinates, of the velocity and electric field vectors are given, respectively, by

$\boldsymbol{v}=v(r) \boldsymbol{e}_{\theta}$,

$\boldsymbol{E}=E \boldsymbol{e}_{r}$,

where $\boldsymbol{e}_{r}, \boldsymbol{e}_{\theta}$ and $\boldsymbol{e}_{z}$ are the unit vectors in the directions $r, \theta$ and $z$, respectively and $E$ is a constant.

The stress components are, by Eqs. (5) and (10)

$$
\begin{aligned}
& T_{r r}=-p+\alpha_{2} E^{2}+\frac{\alpha_{4}}{4} \gamma^{2}+\frac{\alpha_{6}}{2} \gamma^{2} E^{2} \\
& T_{\theta \theta}=-p+\frac{\alpha_{4}}{4} \gamma^{2} \\
& T_{z z}=-p
\end{aligned}
$$


$T_{r \theta}=\frac{1}{2}\left(\alpha_{3}+\alpha_{5} E^{2}\right) \gamma=\mu\left(E^{2}, \gamma^{2}\right) \gamma$

$T_{r z}=T_{\theta z}=0$

in which $\gamma=r(d / d r)(v / r)$, and $p$ is an arbitrary scalar function of position.

Let the velocity in (32) and the stresses in (33) be substituted into the balance of linear momentum. By a standard analysis, it is found that the shear stress field in the fluid is given by

$T_{r \theta}=\frac{M}{2 \pi r^{2}}$

in which $M$ denotes the moment per unit length applied to the inner cylinder. The scalar field $p$ is given by

$p=\alpha_{2} E^{2}+\frac{1}{4} \alpha_{4} \gamma^{2}+\frac{\alpha_{6}}{2} \gamma^{2} E^{2}+\int \frac{1}{\bar{r}}\left[\alpha_{2} E^{2}+\frac{\alpha_{6}}{2} \gamma^{2} E^{2}\right] d \bar{r}+\int \frac{v^{2}}{\bar{r}} d r+$ constant.

A differential equation for $v(r)$ is obtained when the equation for shear stress from (33) is substituted into Eq. (34). The appropriate boundary conditions are

$v\left(R_{i}\right)=\omega_{i} R_{i}, \quad v\left(R_{0}\right)=0$,

where $\omega_{i}$ is the angular velocity of the inner cylinder. Once the velocity field has been determined, the right hand side of Eq. (35) is known and the scalar field $p$ can be evaluated.

The velocity field $v(r)$ will be determined for the $T_{r \theta}-E-\gamma$ relation given in Eq. (23). In doing so, use will be made of the result from $\mathrm{Eq}$. (34) that for each fixed value of $M$ the shear stress decreases monotonically from $M / 2 \pi R_{i}{ }^{2}$ at the inner cylinder to $M / 2 \pi R_{0}{ }^{2}$ at the outer cylinder.

First, let $E=0$ so that, according to Eq. $(23), \sigma_{0}(0)=0$. Then $v(r)$ is given by the well known solution for Couette flow of a Newtonian fluid

$v(r)=\frac{M r}{2 \pi \mu(0)}\left[\frac{1}{R_{0}^{2}}-\frac{1}{r^{2}}\right]$.

The relation between the angular velocity $\omega_{i}$ of the inner cylinder and its driving moment $M$ is obtained by evaluating Eq. (37) at $r=R_{i}$ :

$\omega_{i}=\frac{M}{2 \pi \mu(0)}\left[\frac{R_{i}{ }^{2}-R_{0}{ }^{2}}{R_{i}{ }^{2} R_{0}{ }^{2}}\right]$.

Now let $E$ be a non-zero constant and consider the flow for increasing values of $M$. If $M$ is small enough that $M / 2 \pi R_{i}{ }^{2}<\sigma_{0}(E)$, then $T_{r \theta}<\sigma_{0}(E), R_{i} \leqq r \leqq R_{0}$ and there is no shear flow. Let the value of $E$ be such that $M / 2 \pi R_{i}{ }^{2}>\sigma_{0}(E)$ and $M / 2 \pi R_{0}{ }^{2}<\sigma_{0}(E)$, i.e., $2 \pi R_{i}{ }^{2} \sigma_{0}(E)<M<2 \pi R_{0}{ }^{2} \sigma_{0}(E)$. Then there is some radius $r^{*}(E), R_{i} \leqq r^{*}(E) \leqq R_{0}$, at which

$r^{*}(E)=\left(\frac{M}{2 \pi \sigma_{0}(E)}\right)^{1 / 2}$.

For $R_{i} \leqq r \leqq r^{*}(E)$, there is shear flow, and for $r^{*}(E) \leqq r \leqq R_{0}$ the fluid is rigid. By Eqs. (23), (33) and (34) the velocity field satisfies the differential equations

$$
\begin{aligned}
& \sigma_{0}(E)+\mu(E) r \frac{d}{d r}\left(\frac{v}{r}\right)=\frac{M}{2 \pi r^{2}}, \quad R_{i} \leqq r \leqq r^{*}(E) \\
& \frac{d}{d r}\left(\frac{v}{r}\right)=0, \quad r^{*}(E) \leqq r \leqq R_{0} .
\end{aligned}
$$


The solution of the system of Eqs. (36) and (39) is

$v(r)= \begin{cases}\frac{r}{2 \mu(E)}\left[-\frac{M}{2 \pi r^{2}}+\sigma_{0}(E)+\sigma_{0}(E) \ln \left(\frac{M}{2 \pi \sigma_{0}(E) r^{2}}\right)\right], & R_{i} \leqq r \leqq r^{*}(E) \\ 0 & r^{*}(E) \leqq r \leqq R_{0}\end{cases}$

The moment which is required in order to satisfy the boundary condition at the inner cylinder is determined from

$\omega_{i}=\frac{1}{2 \mu(E)}\left[-\frac{M}{2 \pi R_{i}^{2}}+\sigma_{0}(E)+\sigma_{0}(E) \ln \left(\frac{M}{2 \pi \sigma_{0}(E) R_{i}^{2}}\right)\right]$.

Finally, if $M>2 \pi R_{0}{ }^{2} \sigma_{0}(E)$, then $T_{r \theta}>\sigma_{0}(E), R_{0} \leqq r \leqq R_{i}$. The velocity field satisfies the differential equation

$\sigma_{0}(E)+\mu(E) r \frac{d}{d r}\left(\frac{v}{r}\right)=\frac{M}{2 \pi r^{2}}, \quad R_{i} \leqq r \leqq R_{0}$.

The solution satisfying condition (36) is

$v(r)=\frac{r}{\mu(E)}\left[\frac{M}{4 \pi}\left(\frac{-1}{r^{2}}+\frac{1}{R_{0}^{2}}\right)+\sigma_{0}(E) \ln \left(\frac{R_{0}}{r}\right)\right]$

and the $\omega_{i}-M$ relation is

$\omega_{i}=\frac{1}{\mu(E)}\left[\frac{M}{4 \pi}\left(\frac{R_{i}^{2}-R_{0}^{2}}{R_{i}^{2} R_{0}^{2}}\right)+\sigma_{0}(E) \ln \frac{R_{0}}{R_{i}}\right]$.

The velocity field for all three cases is shown in Fig. 3, and the $M-\omega_{i}$ relation is shown in Fig. 4. In the $M-\omega_{i}$ relation, note the gradual transition to a straight line which is parallel to the one corresponding to $E=0$. This corresponds to the flow described by Eqs. (40) in which there is an inner cylindrical region of shear flow, and a "dead zone". The size of the gap in the flow region is $r^{*}(E)-R_{i}$ and depends on both the applied moment $M$ and the electric field $E$. For fixed $E$, this gap increases with moment $M$. This point is significant in view of the fact in some experimental work, the shear rate of the fluid has been taken as

$v\left(R_{i}\right) /\left(R_{0}-R_{i}\right)=R_{i} \omega_{i} /\left(R_{0}-R_{i}\right)$.

Next, consider the effect of fixing the applied moment $M$ and increasing the electric field $E$. When $E=0$, the fluid is Newtonian and $v(r)$ is given by Eq. (37). Let $E$ increase to a value such that $\sigma_{0}(E)<M / 2 \pi R_{0}{ }^{2}$. Then the shear stress in the fluid exceeds the yield stress at all points in the fluid and $v(r)$ is given by Eq. (43). Let $E$ increase so that $M / 2 \pi R_{0}{ }^{2}$ $<\sigma_{0}(E)<M / 2 \pi R_{i}{ }^{2}$. Then there is a radius

$r^{*}(E)=\left(\frac{M}{2 \pi \sigma_{0}(E)}\right)^{1 / 2}$,

as described in regard to Eqs. (40), which separates the shear flow near the inner cylinder from the "dead zone" near the outer cylinder. As $E$ increases, the interface radius $r^{*}(E)$ decreases. When $\sigma_{0}(E)>M / 2 \pi R_{i}{ }^{2}$, the shear stress in the fluid is everywhere less than the yield stress and the fluid becomes rigid. Finally, as $E$ and $\sigma_{0}(E)$ increase, Eqs. (38), (41) and (44) show that $\omega_{i}$ decreases to zero, as shown in Fig. 5. 

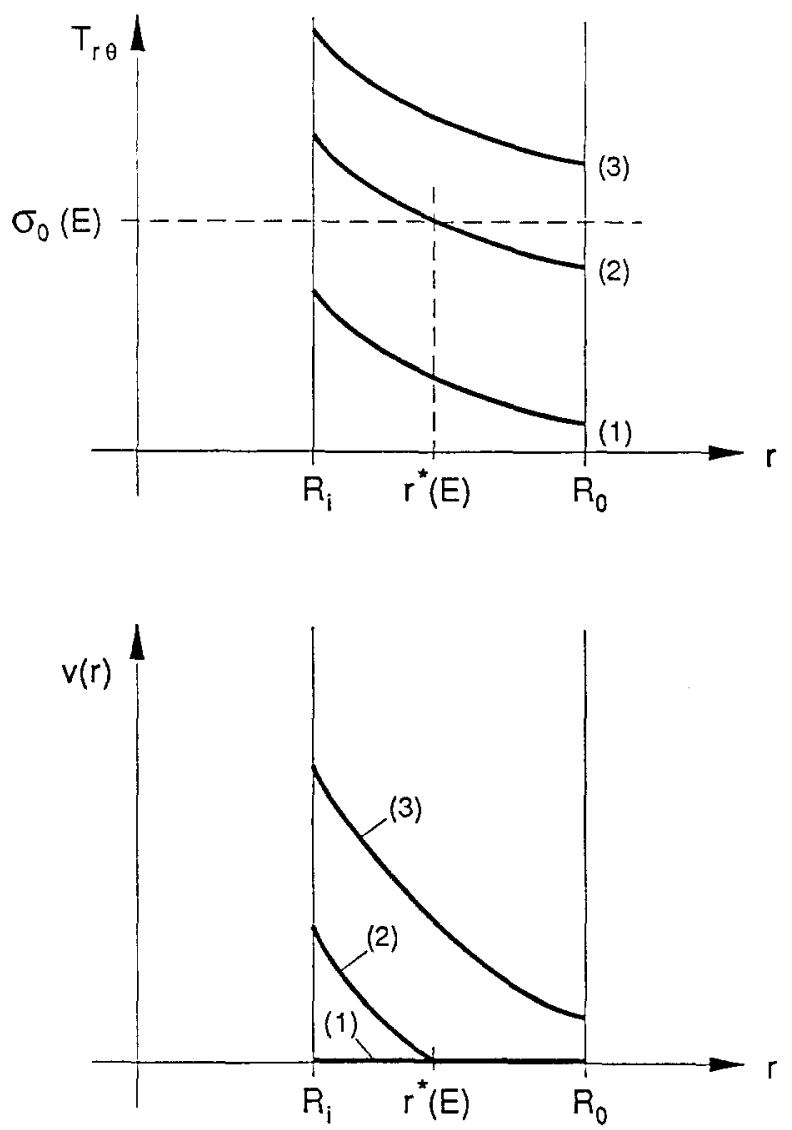

Fig. 3. Conette flow: shear stress and velocity field distributions. The electric field $E$ is fixed and moment $M$ is increasing

Curves (1): $M<2 \pi R_{i}{ }^{2} \sigma_{0}(E)$

Curves (2): $2 \pi R_{i}{ }^{2} \sigma_{0}(E)<M<2 \pi R_{0}{ }^{2} \sigma_{0}(E)$

Curves (3): $M>2 \pi R_{i}{ }^{2} \sigma_{0}(E)$

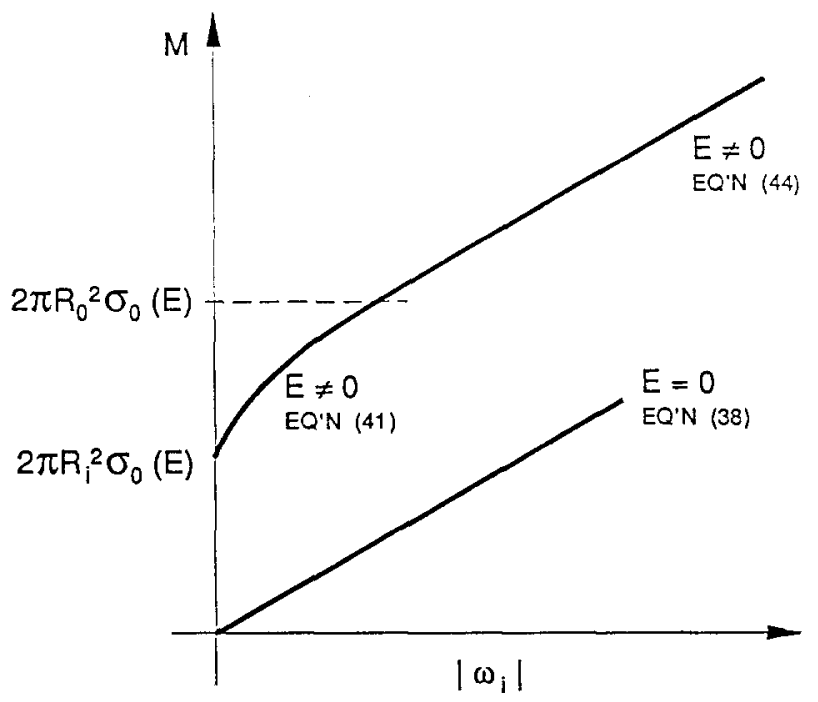

Fig. 4. Couette flow: moment-angular velocity relations for $E=0$ and $E \neq 0$ 


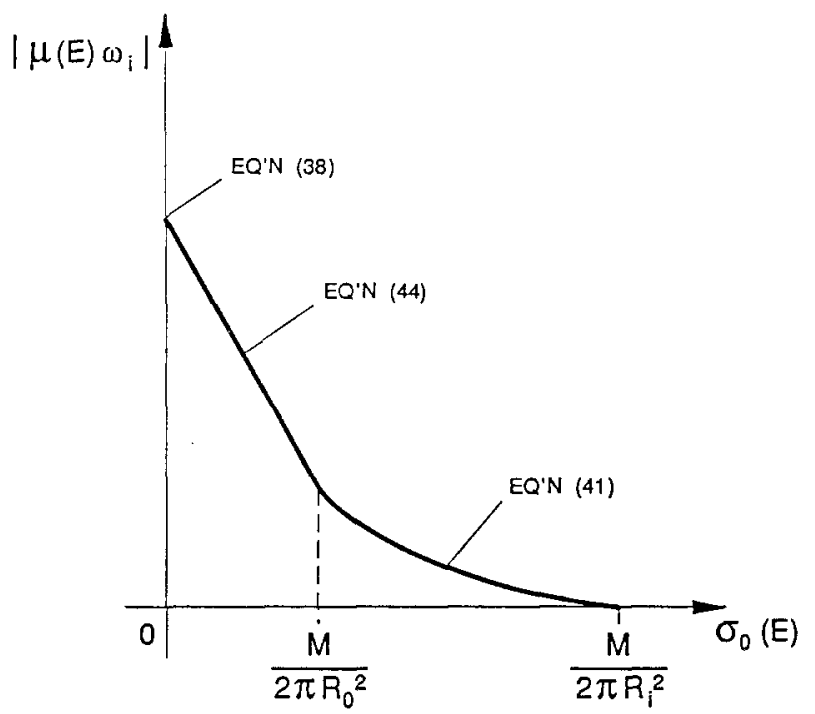

Fig. 5. Couette flow: angular velocity of inner cylinder versus yield stress for fixed moment

According to Eqs. (33), there are normal stress effects which arise in Couette flow due to the presence of the electric field. First, consider the effect on the difference of pressures at the inner and outer cylinders. Let these pressures be denoted, respectively, by $p_{i}=-T_{r r}\left(R_{i}\right)$ and $p_{0}=-T_{r r}\left(R_{0}\right)$. Then from the radial component of the equations of motion

$\frac{d T_{r r}}{d r}+\frac{T_{r r}-T_{\theta \theta}}{r}=-\varrho r \frac{v^{2}}{r}$

the pressure difference is found to be

$p_{0}-p_{i}=\int_{R_{i}}^{R_{0}} \varrho \frac{v^{2}}{r} d r+E^{2} \int_{R_{i}}^{R_{0}} \frac{1}{r}\left(\alpha_{2}+\frac{1}{2} \alpha_{6} \gamma^{2}\right) d r$.

The first integral accounts for the pressure difference which occurs in flows of Newtonian fluids. The second term arises from the presence of the electric field. The deviation from the Newtonian result is quadratic in $E$. On the basis of intuition, one might expect that the electric field induces tension in the fluid in the direction of the electric field vector, and hence that $\alpha_{2}>0$. If $\alpha_{0}$ is positive, or small in magnitude, the electric field $E$ increases this normal stress difference.

There is also a normal stress component $T_{z z}$, an expression for which is given by Eqs. (33) and (35). Its radial variation depends on the material property functions and the electric field. Depending on these, the fluid could display an electro-rheological equivalent of the free surface shape change effects which have been observed in polymerio fluids.

\subsection{Eccentric rotating disc device}

In recent years, there has been a considerable amount of interest in understanding the flow occurring in an eccentric rotating disc device, commonly referred to as an orthogonal rheometer (cf. Rajagopal and Wineman [4]). 


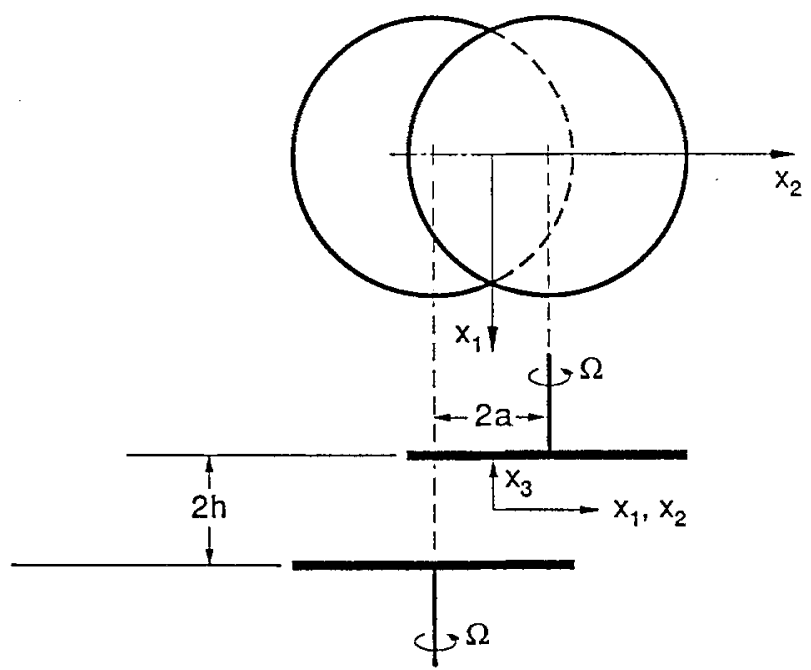

Fig. 6. Eccentric rotating disk device

An idealized eccentric rotating disc device is shown in Fig. 6. In this device, fluid is contained between parallel plates which rotate with the same angular speed about parallel non-coincident axes. The distance between the plates is $2 h$ and the distance between the axes is $2 a$. Measuring the forces and moments which act on the plate one can characterize the material moduli of the fluid that is entrapped in the device. With respect to the Cartesian coordinate axes shown in Fig. 6, the velocity field is assumed to have the form:

$\boldsymbol{v}=v_{1} \boldsymbol{e}_{1}+v_{2} \boldsymbol{e}_{2}$,

where

$v_{1}=-\Omega\left(x_{2}-g\left(x_{3}\right)\right)$,

$v_{2}=\Omega\left(x_{1}-f\left(x_{3}\right)\right)$,

and $\Omega$ denotes the angular velocity of the plates. In this velocity field, the fluid layer at $x_{3}=$ constant undergoes a rigid body rotation about the point $\left(f\left(x_{3}\right), g\left(x_{3}\right)\right)$. The locus of the points about which different $x_{3}=$ constant planes rotate is in general an arbitrary curve in space. It has been shown that (47) is a motion with constant stretch history (cf. Rajagopal [5]), which implies that the eigenvalues of the relative stretch history are constant, the eigendirections changing with time. This fact reduces the equations of motion of general simple fluids to a system of second order non-linear ordinary differential equations with the appropriate number of boundary conditions being provided by the adherence boundary condition.

The standard eccentric rotating disc device is assumed to have been modified so that there is an electric field in the fluid of the form

$\boldsymbol{E}=E \boldsymbol{e}_{3}$,

where $E$ is constant.

The stress components, calculated using Eqs. (5) and (10), are

$T_{11}=-p+\alpha_{4} \frac{\Omega^{2}}{4} g^{\prime 2}$,
$T_{22}=-p+\alpha_{4} \frac{\Omega^{2}}{4} f^{\prime 2}$, 
$T_{33}=-p+\alpha_{2} E^{2}+\left(f^{\prime 2}+g^{\prime 2}\right)\left[\frac{\alpha_{4}}{4} \Omega^{2}+\frac{\alpha_{6}}{2} \Omega^{2} E^{2}\right]$,

$T_{13}=\frac{1}{2}\left(\alpha_{3}+\alpha_{5} E^{2}\right) \Omega g^{\prime}$,

$T_{23}=-\frac{1}{2}\left(\alpha_{3}+\alpha_{5} E^{2}\right) \Omega f^{\prime}$,

$T_{12}=-\alpha_{4} \frac{\Omega^{2}}{4}\left(f^{\prime} g^{\prime}\right)$

where $p$ is an arbitrary scalar. The invariants (6) have the same form as in (14), where now

$\gamma^{2}=\Omega^{2} \varkappa^{2}, \quad \varkappa^{2}=f^{\prime 2}+g^{\prime 2}$.

In view of Eqs. (16) and (50), one can now write

$T_{13}=\mu\left(E^{2}, \gamma^{2}\right) \Omega g^{\prime}$,

$T_{23}=-\mu\left(E^{2}, \gamma^{2}\right) \Omega f^{\prime}$.

Let the velocity field given by Eq. (47) and the stresses given by Eqs. (49) - (51) be substituted into the equations of motion, stated with respect to Cartesian coordinates. The result is

$-\frac{\partial p}{\partial x_{1}}+\frac{\partial}{\partial x_{3}} T_{13}=-\varrho \Omega^{2}\left(x_{1}-f\right)$,

$-\frac{\partial p}{\partial x_{2}}+\frac{\partial}{\partial x_{3}} T_{23}=-\varrho \Omega^{2}\left(x_{2}-g\right)$,

$-\frac{\partial p}{\partial x_{3}}+\frac{\partial}{\partial x_{3}} \hat{T}_{33}=0$,

where

$\hat{T}_{33}=T_{33}+p$.

Application of the curl operation to these equations eliminates the scalar $p$ and leads to

$\frac{d}{d x_{3}} T_{13}=\varrho \Omega^{2} f+q$,

$\frac{d}{d x_{3}} T_{23}=\varrho \Omega^{2} g+s$,

and $s$ and $q$ are constants. Equations $(53.1,2)$ together with Eqs. $(50)$ and $(51)$ are a system of two differential equations for $f\left(x_{3}\right)$ and $g\left(x_{3}\right)$. The existence of solutions to these equations which are associated with $q \neq 0$ and $s \neq 0$, and which are not symmetric about the plane $x_{3}=$ constant, are discussed by Rajagopal, Renardy, Renardy and Wineman [6] for a class of models for polymer fluids. We shall confine attention to the case $q=0$ and $s=0$, which implies symmetry about the midplane.

The appropriate boundary conditions on $f\left(x_{3}\right)$ and $g\left(x_{3}\right)$ arise from the fact that $\left(f\left(x_{3}\right)\right.$, $\left.g\left(x_{3}\right)\right)$ are the coordinates of the center of rotation of the layer at coordinate $x_{3}$ and the fact that the fluid adheres to the rotating plates. The boundary conditions are therefore

$f(h)=f(-h)=0, \quad g(h)=a, \quad g(-h)=-a$. 
The scalar $p$ can be easily obtained and is given by the expression

$p=\frac{\Omega^{2}}{2}\left(x_{1}{ }^{2}+x_{2}{ }^{2}\right)+\hat{T}_{33}+$ constant.

Let the following dimensionless variables be introduced:

$\hat{x}_{3}=x_{3} / h, \quad \hat{f}=f / a, \quad \hat{g}=g / a$,

$\varkappa=\frac{a}{h} \hat{\varkappa}, \quad \hat{\varkappa}^{2}=\left[\left(\frac{d \hat{f}}{d \hat{x}_{3}}\right)^{2}+\left(\frac{d \hat{g}}{d \hat{x}_{3}}\right)^{2}\right]$.

Also let $\mu_{0}$ be a characteristic viscosity and by Eq. (50), define $\hat{\mu}=\mu / \mu_{0}$. The boundary value problem (53) and (54) becomes

$\frac{d}{d \hat{x}_{3}}\left[\hat{a}\left(E^{2},\left(\frac{\Omega a}{h}\right)^{2} x^{2}\right) \frac{d \hat{g}}{d \hat{x}_{3}}\right]=\Lambda^{2} \hat{f}$,

$\frac{d}{d \hat{x}_{3}}\left[\hat{\mu}\left(E^{3},\left(\frac{\Omega a}{h}\right)^{2} \hat{\varkappa}^{2}\right) \frac{d \hat{f}}{d \hat{x}_{3}}\right]=-\Lambda^{2} \hat{g}$,

$\hat{f}(1)=\hat{f}(-1)=0, \quad g(1)=1, \quad g(-1)=-1$,

where $\Lambda$ is a dimensionless parameter defined by

$\Lambda^{2}=\frac{\varrho \Omega h^{2}}{\mu_{0}}$,

and represents a Reynold's number.

We confine attention to the case in which $|\Lambda| \ll 1$. In this case, Eq. (57) admits the approximate solution

$f\left(x_{3}\right)=0, \quad g\left(x_{3}\right)=\frac{a}{h} x_{3}$.

Then $f^{\prime}\left(x_{3}\right)=0, g^{\prime}\left(x_{3}\right)=a / h, \gamma^{2}=(\Omega a / h)^{2}$ and the shear stress field is uniform. The tangential forces on plates of area $A$ are given by

$T_{1}=T_{13} A=\mu\left(E^{2},\left(\frac{\Omega a}{h}\right)^{2}\right)\left(\frac{\Omega a}{h}\right) A$,

$T_{2}=T_{23} A=0$.

Measurement of these forces provides a means of determining $\mu$. It should also be noted that if the angular velocity is held fixed, these forces will vary with the magnitude of $E$.

\subsection{Poiseuille flow between the annulus of two cylinders}

Consider the pressure induced flow between the annulus of two co-axial cylinders which are fixed in space with the axis aligned along the $z$-coordinate direction, the outer cylinder of radius $R_{0}$ and the inner cylinder of radius $R_{i}$, with $R_{0}>R_{i}$. Suppose that the cylinders are infinitely long and that the flow is fully developed. We shall assume the velocity field and the electrical field to have the following form:

$\boldsymbol{u}=u(r) \boldsymbol{e}_{z}$ and $\boldsymbol{E}=\boldsymbol{E}_{r}$. 
It follows from (61) and (5) that

$T_{r r}=-p+\alpha_{2} E^{2}+\frac{\alpha_{4}}{4}\left(u^{\prime}\right)^{2}+\frac{\alpha_{6}}{2} E^{2}\left(u^{\prime}\right)^{2}$,

$T_{\theta \theta}=-p$,

$T_{z z}=-p+\frac{\alpha_{4}}{4}\left(u^{\prime}\right)^{2}$,

$T_{r z}=\frac{\alpha_{3}}{2} u^{\prime}+\frac{\alpha_{5}}{2} E^{2} u^{\prime}$,

all the other components of the stress being zero.

The equations of motion reduce to

$-\frac{\partial p}{\partial r}+\frac{d}{d r}\left[\alpha_{2} E^{2}+\alpha_{4}\left(u^{\prime}\right)^{2}+2 \alpha_{6} E^{2}\left(u^{\prime}\right)^{2}\right]+\frac{1}{r}\left[\alpha_{2} E^{2}+\alpha_{1}\left(u^{\prime}\right)^{2}+2 \alpha_{6} E^{2}\left(u^{\prime}\right)^{2}\right]=0$,

$-\frac{\partial p}{\partial \theta}=0$

and

$-\frac{\partial p}{\partial z}+\frac{1}{2 r} \frac{d}{d r}\left[r\left(\alpha_{3} u^{\prime}+\alpha_{5} E^{2} u^{\prime}\right)\right]=0$.

Equations (63.1), (63.2) imply that

$\frac{1}{r} \frac{d}{d r}\left[r\left(\alpha_{3}+\alpha_{5} E^{2}\right) u^{\prime}\right]=C=-\frac{\partial p}{\partial z}=$ constant.

Also $C>0$, because we expect the flow to take place in the direction of decreasing pressure.

Thus, it follows from (62.4) and (64) that

$T_{r z}=\frac{C r}{2}+\frac{D}{r}$

where $D$ is a constant. When $E=0$ and $\alpha_{3}=\mu=$ constant, (64) reduces to the equation governing the classical linearly viscous fluid. Rewriting (65) in terms of the shear viscosity function we find

$\mu\left(E^{2}, \gamma^{2}\right) \gamma=\frac{C r}{2}+\frac{D}{r}$

where $\gamma=u^{\prime}$. In general (66) is a highly non-linear equation for $u^{\prime}$ which can only be solved numerically. The appropriate boundary conditions are

$u\left(R_{i}\right)=0=u\left(R_{0}\right)$.

Let us consider a relation between $T_{r z}, E$, and $\gamma$ of the form

$T_{r z}=\left\{\begin{array}{cc}\sigma_{0}(E)+\mu(E) \gamma & \gamma>0 \\ 0 & \gamma=0 \\ -\sigma_{0}(E)+\mu(E) \gamma & \gamma<0\end{array}\right.$

where $\sigma_{0}(0)=0, \sigma_{0}(E)$ and $\mu(E)$ increase monotonically with $E$. As in the case of flow between parallel plates, the response is similar to that of a Bingham material, with yield stress $\sigma_{0}(E)$, and we shall not go into a detailed repetitious discussion here. 
Let us now determine $u(r)$. Let us suppose that the pressure gradient is such that the shear stress exceeds the yield stress everywhere then, it follows from (65) and (68) that

$u(r)=\frac{1}{\mu(E)}\left[\frac{C r^{2}}{4}+D \log r-\sigma_{0}(E) r+K\right]$,

where $D$ and $K$ are constants which are to be determined by using the boundary conditions $(67), \sigma_{0}(E)$ is a specified number, and $C$ is the negative of the applied pressure gradient in the $z$-direction. It follows from (67) and (69) that

$-D=\frac{\frac{C}{4}\left(R_{i}\right)^{2}\left(\alpha^{2}-1\right)+R_{i}(\alpha-1) \sigma_{0}(E)}{\ln \alpha}$

and

$K=\frac{C R_{i}^{2}}{4}\left[\left(\alpha^{2}-1\right) \frac{\ln R_{i}}{\ln \alpha}-1\right]+\sigma_{0}(E) R_{i}\left[1+(\alpha-1) \frac{\ln R_{i}}{\ln \alpha}\right]$

where

$\alpha=\frac{R_{0}}{R_{i}}$.

By allowing $R_{i} \rightarrow 0$ and requiring that the flow field be bounded, we would recover the result for Poiseuille flow in a pipe, the axis of the pipe coinciding with a wire of infinitesimal thickness.

If the shear stress does not exceed the yield stress everywhere, and this could be the case if the applied electric field is very strong and/or the applied pressure gradient is weak, we will have "dead zones" like those discussed in the Section of flow between parallel plates. We shall not get into a detailed discussion of the same here.

\section{Acknowledgement}

The authors thank Prof. Filisko for insightful discussion on this subject.

\section{References}

[1] Truesdell, C., Toupin, R.: The classical field theories. Handbuch der Physik, Bd. IV/1. Berlin: Springer 1960.

[2] Spencer, A. J. M.: In: Continuum physies (Eringen, A. C., ed.), vol. 3. New York: Academic Press 1975.

[3] Filisko, F.: Private communication.

[4] Rajagopal, K. R., Wineman, A. S.: Flow of a BKZ fluid in an orthogonal rheometer. J. Rheol. 27, 509-516 (1983).

[5] Rajagopal, K. R.: On the flow of a simple fluid in an orthogonal rheometer. Arch. Rational Mech. Anal. 79, 39-47 (1982).

[6] Rajagopal, K. R., Renardy, M., Renardy, Y., Wineman, A. S.: Flow of viscoelastic fluids between plates rotating about distinct axes. Rheol. Acta 25, 459-467 (1986).

Authors' addresses: K. R. Rajagopal, Department of Mechanical Engineering, University of Pittsburgh, Pittsburgh, PA 15261, U.S.A., and A. S. Wineman, Department of Mechanical Engineering and Applied Mechanics, University of Michigan, Ann Arbor, MI 48109, U.S.A. 